\title{
Outcome of Coil Embolization for Original Symptomatic Unruptured Cerebral Aneurysms
}

Shingo Nishihiro, Kenji Sugiu, Tomohito Hishikawa, Masafumi Hiramatsu, Naoya Kidani, Yu Takahashi, and Isao Date

Purpose: We examined the outcome of coil embolization for symptomatic unruptured cerebral aneurysms, and investigated factors contributing to symptom relief after surgery.

Methods: We retrospectively reviewed 19 patients who underwent coil embolization between January 2008 and April 2016.

Results: After surgery, symptoms reduced in 13 patients (68\%), but not in $6(32 \%)$. In the improved group, the mean duration of disease was significantly shorter than in the unimproved group (1.3 vs. 16.2 months, respectively, $p<0.05$ ). There was a significant reduction in the aneurysmal size in the former (mean rate of reduction: $3.51 \mathrm{~mm}$ ), whereas there was an increase in the latter (mean increase: $1.63 \mathrm{~mm})(p<0.05)$.

Conclusion: Early therapeutic intervention and a postoperative reduction in the aneurysmal size may be important to achieve the relief of symptoms of symptomatic unruptured cerebral aneurysms after surgery.

Keywords > coil embolization, symptomatic unruptured aneurysm

\section{Introduction}

Unruptured cerebral aneurysms may compress the peripheral cerebral nerves/parenchyma through aneurysmalenlargement-related mass effects, causing symptoms. ${ }^{1}{ }^{1} \mathrm{To}$ relieve symptoms of symptomatic unruptured cerebral aneurysms, it is necessary to reduce compression of the peripheral cerebral tissue. Previous studies indicated that coil embolization did not reduce the mass effects due to the use of fulfilled coils, but patients with symptom relief have also been reported. ${ }^{2-8)}$

We examined the results of coil embolization for symptomatic unruptured cerebral aneurysms in our department

Department of Neurological Surgery, Okayama University Graduate School of Medicine, Okayama, Okayama, Japan

Received: April 13, 2017; Accepted: October 3, 2017

Corresponding author: Kenji Sugiu. Department of Neurological Surgery, Okayama University Graduate School of Medicine, 2-5-1 Shikata-cho, Kita-ku, Okayama, Okayama 700-8558, Japan Email:ksugiu@md.okayama-u.ac.jp

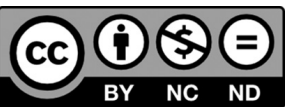

This work is licensed under a Creative Commons Attribution-NonCommercialNoDerivatives International License.

(C)2018 The Japanese Society for Neuroendovascular Therapy and factors involved in symptom relief after surgery, and reviewed the literature.

\section{Subjects and Methods}

Of patients who underwent coil embolization for symptomatic unruptured cerebral aneurysms in our department between January 2008 and April 2016, the subjects were 19 , excluding those undergoing additional treatment. We examined the localization of aneurysms, aneurysmal size before and after surgery, size changes after surgery, presence or absence of thrombosis within aneurysms, preoperative symptoms, duration of disease, presence or absence of symptom relief after surgery, and clarified factors contributing to symptom relief after surgery.

In each patient, clinical symptoms were evaluated by the same neurosurgeon before and after surgery. Preoperative evaluation was conducted immediately before treatment. As postoperative evaluation, symptoms on the final follow-up were compared with preoperative neurologic symptoms, and assessed using 3 grades: "improvement," "no change," and "exacerbation."

For image assessment, the maximum diameter of an aneurysm, involving the site of thrombosis and coil mass, 
Table 1 Characteristics of 19 patients with symptomatic aneurysm

\begin{tabular}{lc} 
Characteristics & $\mathrm{n}(\%)$ \\
Sex & \\
Male & $6(32)$ \\
Female & $13(68)$ \\
Age (year) & $67.1 \pm 11.1$ \\
Size of aneurysm (mm) & $20.8 \pm 8.8$ \\
Aneurysm location & \\
IC-cavernous & $8(42)$ \\
IC-PC & $5(26)$ \\
VA & $3(16)$ \\
BA & $2(11)$ \\
IC-PPTA & $1(5)$ \\
Partially thrombosed aneurysm & $10(53)$ \\
Symptoms & \\
Oculomotor nerve palsy & $12(50)$ \\
Abducens nerve palsy & $6(25)$ \\
Hemiparesis & $2(8)$ \\
Visual disturbance & $1(4)$ \\
Cerebellar ataxia & $1(4)$ \\
Hypoglossal nerve palsy & $1(4)$ \\
Sensory disturbance & $1(4)$ \\
Interval between onset and treatment (months) & $6 \pm 14$ \\
Treatment & \\
Coil embolization & $16(84)$ \\
Parent artery occlusion & $3(16)$ \\
\hline BA: basilar arery IC-PC: inter & \\
\hline
\end{tabular}

BA: basilar artery; IC-PC: internal carotid-posterior communicating; PPTA: persistent primitive trigeminal artery; VA: vertebral artery

was measured using source images on time-of-flight MRI, and postoperative images on the final follow-up were compared with preoperative ones.

To compare the values between two groups, statistical analysis was performed using Student's t-test and Fisher's exact test. A p value of 0.05 was regarded as significant.

\section{Results}

The 19 subjects with symptomatic unruptured cerebral aneurysms consisted of 6 males and 13 females, with a mean age of $67.1 \pm 11.1$ years (34-88 years). Aneurysms were localized in the cavernous portion in eight patients $(42 \%)$, accounting for the highest percentage. The mean preoperative aneurysmal size was $20.8 \pm 8.8 \mathrm{~mm}(10-44.5 \mathrm{~mm})$. The most frequent preoperative symptom was oculomotor nerve palsy in 12 patients $(50 \%)$. The mean duration of disease was $6 \pm 14$ months ( 5 days to 5 years and 4 months). As endovascular treatment procedures, intraaneurysmal embolization was performed for 16 patients (stent-assisted coil embolization: 10 patients), and parent vessel occlusion for three patients (Table 1).

The mean follow-up period was $27.7 \pm 22.4$ months (1 month to 8 years and 6 months). The mean postoperative aneurysmal size was $18.8 \pm 8.5 \mathrm{~mm}(7.6-44.1 \mathrm{~mm})$, showing a tendency of reduction compared with the preoperative value, with no significant difference $(p=0.117)$. After surgery, the symptoms reduced in 13 patients $(68 \%)$, whereas "no change" or "exacerbation" was noted in $6(32 \%)$. There were no marked differences in the interval until recovery or degree of symptom relief among the neurologic symptoms. The preoperative aneurysmal size, duration of disease, rate of change in the aneurysmal size (postoperative value-preoperative value), and partially thrombosed aneurysms were compared between patients with and without symptom relief after surgery. In the former, the preoperative aneurysmal size was slightly smaller than in the latter $(18.8 \pm 7.2$ vs. $25.1 \pm 10.2 \mathrm{~mm}$, respectively, $p=0.166$ ). The mean duration of disease was significantly shorter in the former $(1.3 \pm 1.0$ vs. $16.2 \pm 21.6$ months, respectively, $\mathrm{p}<0.05)$. Concerning the rate of change in the aneurysmal size, the mean postoperative aneurysmal size in the former was significantly reduced (mean rate of change: $-3.51 \pm 5.03$ vs. $1.63 \pm 2.18 \mathrm{~mm}$, respectively, $\mathrm{p}<0.05)$. Partially thrombosed aneurysms were present in $8(62 \%)$ of 13 patients with symptom relief after surgery and in $2(33 \%)$ of 6 without it; they were more frequent in patients with symptom relief (Fisher's exact test, $p=0.252$ ) (Table 2). 
Table 2 The factors that contribute to the recovery of symptoms after the treatment

\begin{tabular}{lccc} 
Factors & Improved & Unchanged & $P$ value \\
Preoperative aneurysmal size $(\mathrm{mm})$ & $18.8 \pm 7.2$ & $25.1 \pm 10.2$ & 0.166 \\
Interval between onset and treatment (months) & $1.3 \pm 1.0$ & $16.2 \pm 21.6$ & $<0.05$ \\
Change of aneurysmal size $(\mathrm{mm})$ & $-3.51 \pm 5.03$ & $1.63 \pm 2.18$ & $<0.05$ \\
Partially thrombosed aneurysm, $\mathrm{n}(\%)$ & $8(62)$ & $2(33)$ & 0.252 \\
\hline
\end{tabular}
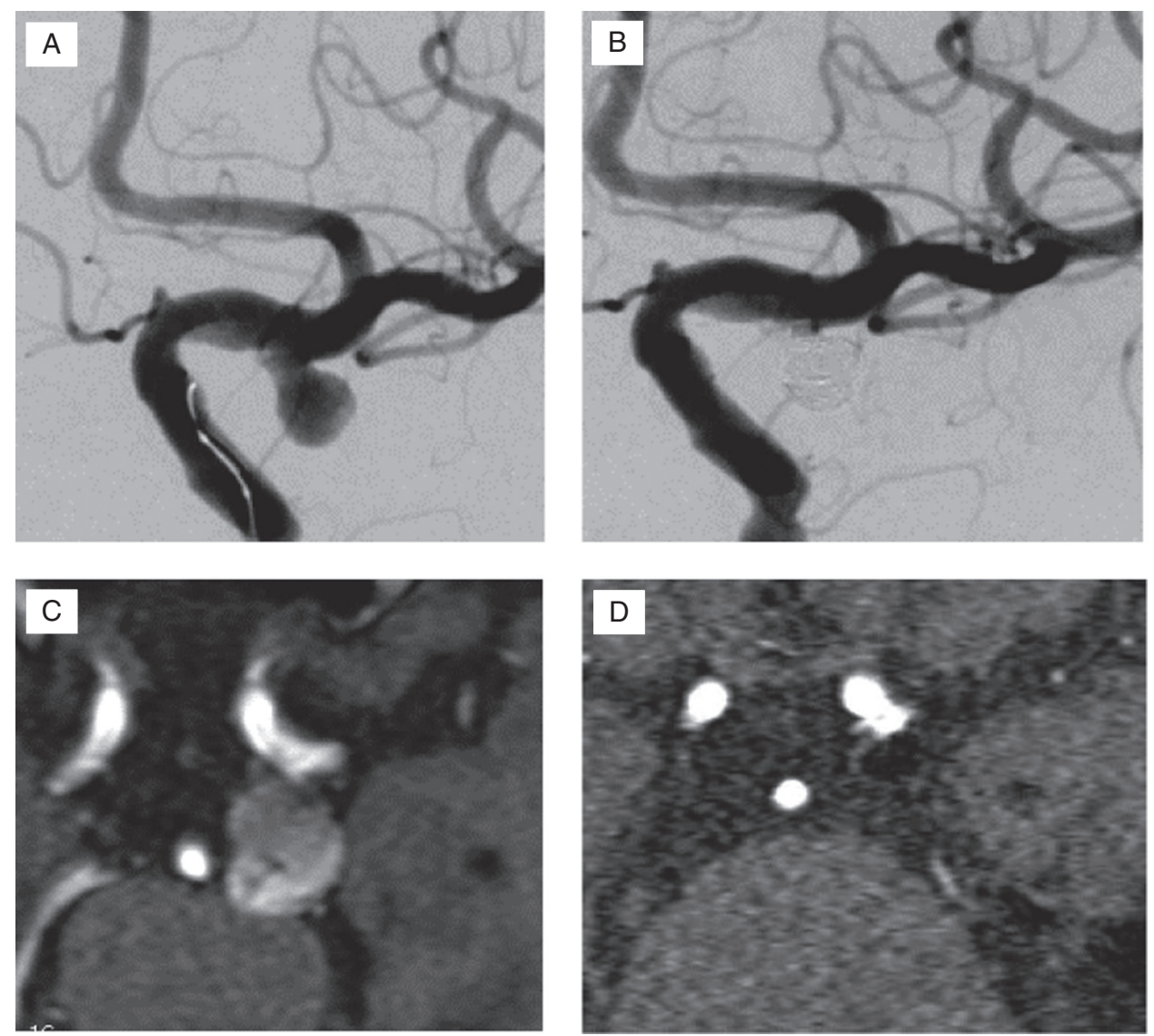

Fig. 1 The partially thrombosed IC-PC aneurysm presenting oculomotor nerve palsy. (A) Angiography before the treatment. (B) Angiography immediately after the treatment. Angiography showed the complete obliteration with balloon remodeling technique. (C) MRA (time-of-flight) before the treatment. (D) MRA (time-of-flight) at 31 months after the treatment. The size of aneurysm was remarkably decreased after the treatment. IC-PC: internal carotid-posterior communicating

\section{Case Presentations}

\section{Case 1 (Fig. 1)}

An 80-year-old male with a left internal carotid-posterior communicating artery aneurysm (maximum diameter: $15 \mathrm{~mm}$, partially thrombosed aneurysm) presented with oculomotor nerve palsy. The duration of disease was 2 months. Using the balloon remodeling technique, coil embolization was performed. After surgery, there was a reduction in the aneurysmal size. After 3 months, complete recovery from oculomotor nerve palsy was achieved.

\section{Case 2 (Fig. 2)}

A 66-year-old female with a left internal carotid-cavernous aneurysm (maximum diameter: $14 \mathrm{~mm}$, partially thrombosed aneurysm) presented with oculomotor nerve palsy. The duration of disease was 1 month. Stent-assisted coil embolization was performed. After surgery, there was no reduction in the aneurysmal size, but complete recovery from oculomotor nerve palsy was achieved after 4 months.

\section{Case 3 (Fig. 3)}

A 66-year-old female with a left internal carotid-posterior communicating artery aneurysm (maximum diameter: $29 \mathrm{~mm}$, 

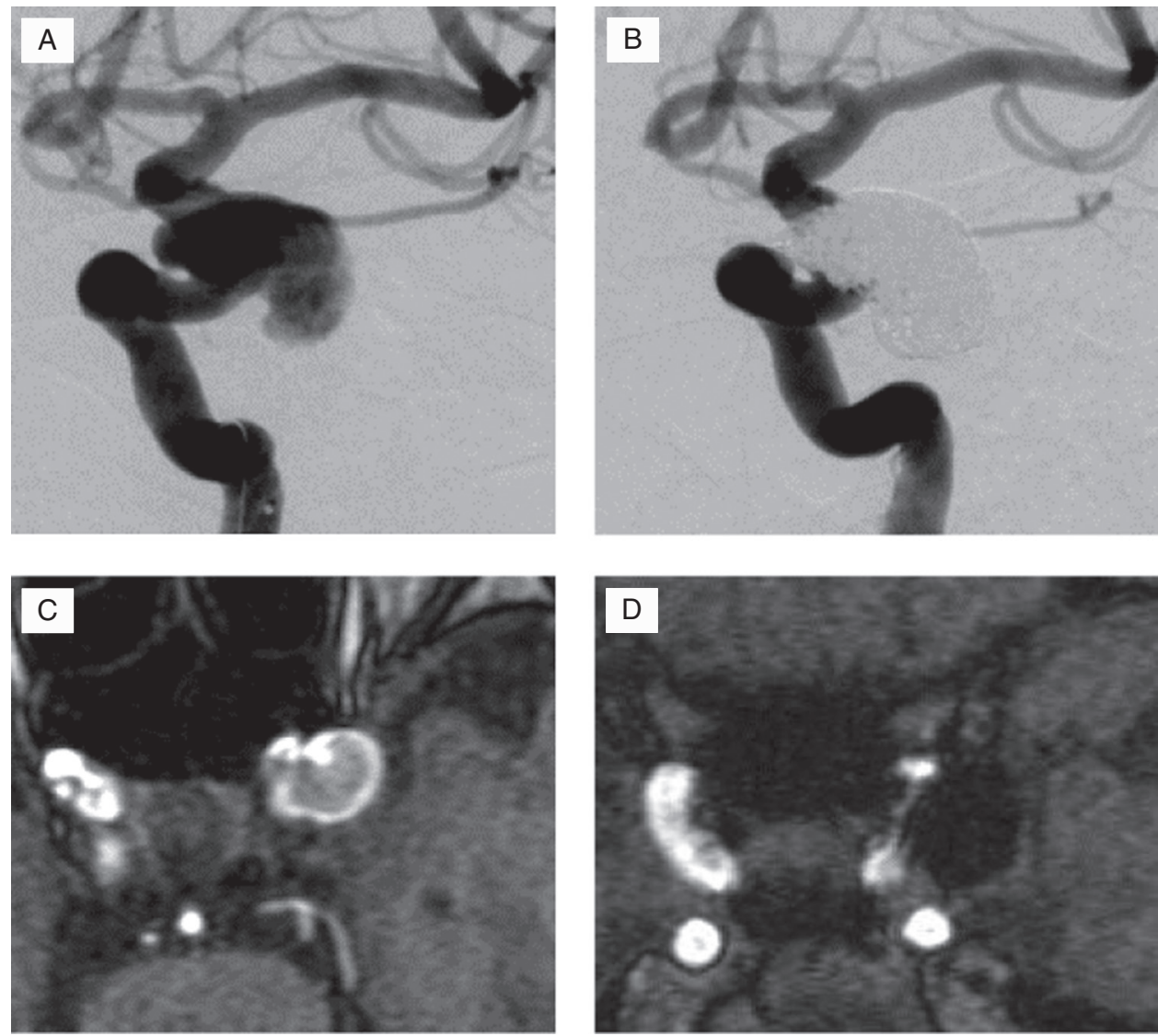

Fig. 2 The partially thrombosed IC-cavernous aneurysm presenting oculomotor nerve palsy. (A) Angiography before the treatment. (B) Angiography immediately after the treatment. Angiography showed the complete obliteration with stent-assisted coil embolization. (C) MRA (time-of-flight) before the treatment. (D) MRA (time-of-flight) at 30 months after the treatment. The size of aneurysm was not decreased after the treatment. IC: internal carotid

partially thrombosed aneurysm) presented with left visual disturbance (only light perception). The duration of disease was 9 months. Stent-assisted coil embolization was performed, but there was no reduction in the aneurysmal size after surgery, and symptom relief was not achieved.

\section{Discussion}

Unruptured cerebral aneurysms, with increase in size, may cause cranial nerve palsy or symptoms due to brainstem compression. Of unruptured cerebral aneurysms, approximately $8 \%$ reportedly induce symptoms due to mass effect. $\left.{ }^{9}\right)$

Following surgical interventions, the relief of symptoms caused by unruptured aneurysms are contributed by the decreased mass effect due to size reduction and loss pulsation. ${ }^{5,7)}$ With respect to an improvement in mass effects, such effects can be directly reduced on clipping under craniotomy, but not on coil embolization. Therefore, symptom relief is less marked than on clipping. ${ }^{7)}$
However, some studies indicated that symptom relief was achieved after surgical intervention even in patients who underwent coil embolization for symptomatic cerebral aneurysms. ${ }^{2-8)}$ In this study, there was also a reduction in the aneurysmal size after surgery although there was no significant difference in comparison with the preoperative value; the improvement of mass effects may not have been marked. However, several studies reported that the loss of aneurysmal pulsation after surgery contributed to symptom relief although mass effects remained after coil embolization. ${ }^{10,11)}$ In this study, symptom relief was achieved in the absence of changes in the aneurysmal size after surgery in some patients; not only the reduction of mass effects but also the loss of aneurysmal pulsation may be closely involved in symptom relief early after coil embolization.

As factors contributing to symptom relief after surgery, the age, presence or absence of diabetes mellitus, severity of symptoms, and duration of disease have been 

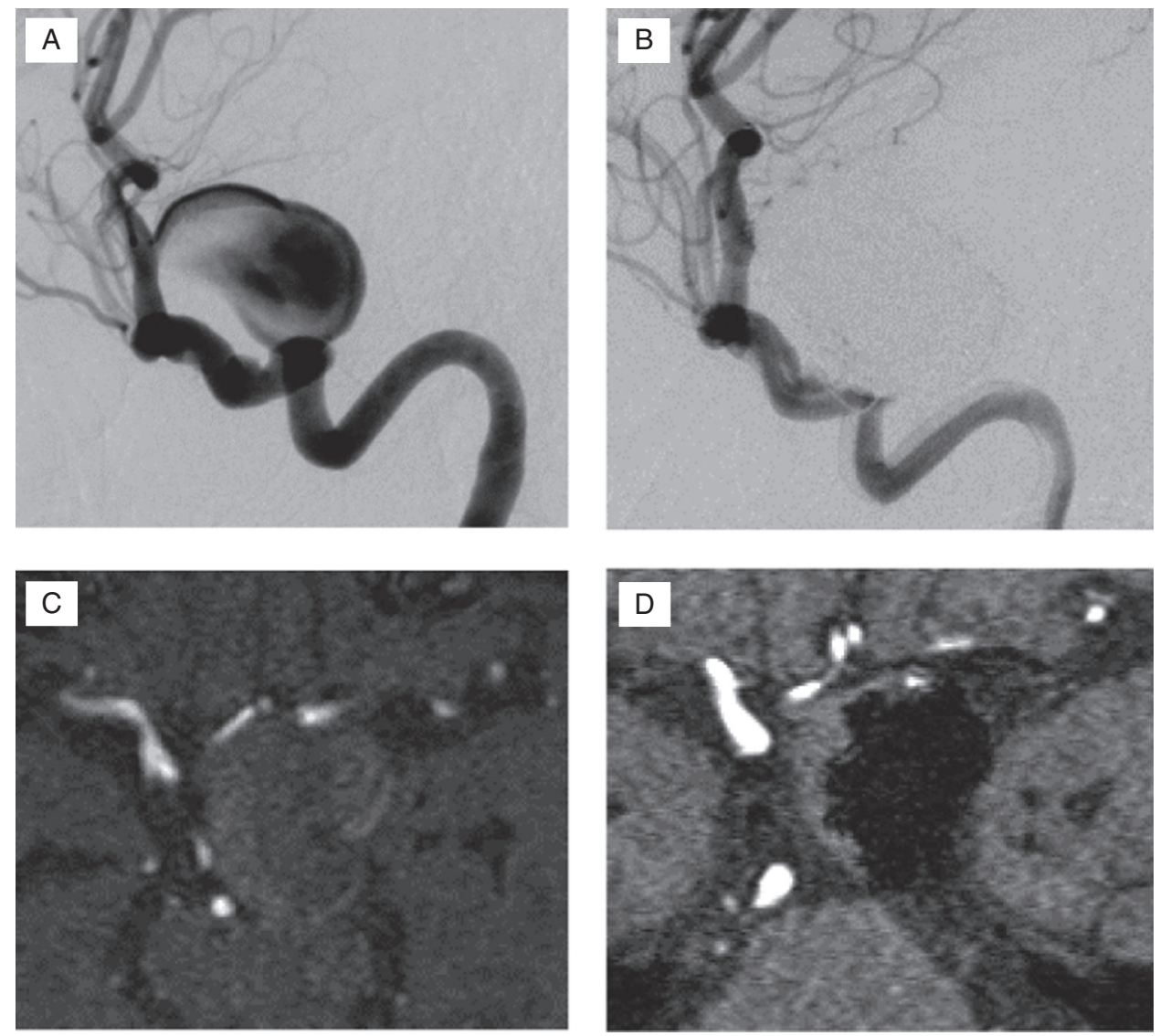

Fig. 3 The partially thrombosed IC-PC aneurysm presenting visual disturbance. (A) Angiography before the treatment. (B) Angiography immediately after the treatment. Angiography showed the complete obliteration with stent-assisted coil embolization. (C) MRA (time-of-flight) before the treatment. (D) MRA (time-of-flight) at 34 months after the treatment. The size of aneurysm was not decreased after the treatment. IC-PC: internal carotid-posterior communicating

reported. ${ }^{4-6,12,13)}$ However, a study reported that the preoperative aneurysmal size did not influence the presence or absence of symptom relief. ${ }^{9)}$ In this study, the preoperative aneurysmal size in patients with symptom relief after surgery was smaller than in those without it, but there was no significant difference. The duration of disease was significantly shorter in the former, suggesting that therapeutic intervention early after onset contributes to symptom relief after surgery. As demonstrated in Case 3, there are some cases in which symptom relief was not achieved after surgery, with a long duration of disease, whereas treatment early after onset may relieve symptoms even in patients without a reduction in the aneurysmal size after surgery, as demonstrated in Case 2. A study emphasized that a delay in the start of treatment led to a poor prognosis; ${ }^{10)}$ therapeutic intervention early after onset will be recommended.

Thrombosed aneurysms may be reduced in the process of thrombus absorption. ${ }^{2,14)}$ In this study, there was a tendency of reduction in size with thrombosed aneurysms after surgery, and thrombosed aneurysms were more frequent in patients with symptom relief after surgery. Thrombosed aneurysms may not influence coil mass effects, and a reduction in the aneurysmal size after surgery may have contributed to symptom relief due to the improvement of mass effects. However, intra-aneurysmal embolization for partially thrombosed aneurysms may lead to recanalization related to a coil mass submerged into the thrombus; recurrence must be considered. ${ }^{14)}$

Considering the mechanism of symptom appearance related to symptomatic cerebral aneurysms, the placement of flow diverter will disperse the aneurysmal pulsation and promote the thrombosis, and may contribute to symptom relief as a consequence. ${ }^{15,16)}$ According to a study, ${ }^{17)}$ there were no changes in the aneurysmal size after the insertion of a flow diverter, and the mass effects remained, whereas symptom relief was achieved; a reduction in aneurysmal pulsation through flow diversion effects may have contributed to symptom relief. 
As symptomatic cerebral aneurysms exhibit mass effects, most aneurysms are relatively large; treatment may be difficult. Coil embolization for such large aneurysms may more frequently result in incomplete occlusion, leading to recurrence. Therefore, as treatment for large symptomatic cerebral aneurysms, flow-diverter insertion may be effective from the viewpoints of the improvement of mass effects and loss of aneurysmal pulsation.

As the limitations of this study, the number of patients was small, and the results were based on univariate analysis; these limitations must be considered.

\section{Conclusion}

Early therapeutic intervention and a reduction in the aneurysmal size after surgery may be important to achieve symptom relief related to coil embolization for symptomatic unruptured cerebral aneurysms.

\section{Disclosure Statement}

There is no conflict of interest for the main author and coauthors.

\section{References}

1) Date I: Symptomatic unruptured cerebral aneurysms: features and surgical outcome. Neurol Med Chir (Tokyo) 2010; 50: 788-799.

2) Halbach VV, Higashida RT, Dowd CF, et al: The efficacy of endosaccular aneurysm occlusion in alleviating neurological deficits produced by mass effect. J Neurosurg 1994; 80: 659-666.

3) Malisch TW, Guglielmi G, Viñuela F, et al: Unruptured aneurysms presenting with mass effect symptoms: response to endosaccular treatment with Guglielmi detachable coils. Part I. Symptoms of cranial nerve dysfunction. J Neurosurg 1998; 89: 956-961.

4) Stiebel-Kalish H, Maimon S, Amsalem J, et al: Evolution of oculomotor nerve paresis after endovascular coiling of posterior communicating artery aneurysms: a neuroophthalmological perspective. Neurosurgery 2003; 53: 1268-1273; discussion 1273-1274.

5) Santillan A, Zink WE, Knopman J, et al: Early endovascular management of oculomotor nerve palsy associated with posterior communicating artery aneurysms. Interv Neuroradiol 2010; 16: 17-21.
6) Ko JH, Kim YJ: Oculomotor nerve palsy caused by posterior communicating artery aneurysm: evaluation of symptoms after endovascular treatment. Interv Neuroradiol 2011; 17: 415-419.

7) Tan H, Huang G, Zhang T, et al: A retrospective comparison of the influence of surgical clipping and endovascular embolization on recovery of oculomotor nerve palsy in patients with posterior communicating artery aneurysms. Neurosurgery 2015; 76: 687-694; discussion 694.

8) Mino M, Yoshida M, Morita T, et al: Outcomes of oculomotor nerve palsy caused by internal carotid artery aneurysm: comparison between microsurgical clipping and endovascular coiling. Neurol Med Chir (Tokyo) 2015; 55: 885-890.

9) Solomon RA, Fink ME, Pile-Spellman J: Surgical management of unruptured intracranial aneurysms. J Neurosurg 1994; 80: 440-446.

10) Ahn JY, Han IB, Yoon PH, et al: Clipping vs coiling of posterior communicating artery aneurysms with third nerve palsy. Neurology 2006; 66: 121-123.

11) Mansour N, Kamel MH, Kelleher M, et al: Resolution of cranial nerve paresis after endovascular management of cerebral aneurysms. Surg Neurol 2007; 68: 500-504; discussion 504.

12) Hanse MC, Gerrits MC, van Rooij WJ, et al: Recovery of posterior communicating artery aneurysm-induced oculomotor palsy after coiling. AJNR Am J Neuroradiol 2008; 29: 988-990.

13) Yanaka K, Matsumaru Y, Mashiko R, et al: Small unruptured cerebral aneurysms presenting with oculomotor nerve palsy. Neurosurgery 2003; 52: 553-557; discussion $556-557$.

14) Ferns SP, van Rooij WJ, Sluzewski M, et al: Partially thrombosed intracranial aneurysms presenting with mass effect: long-term clinical and imaging follow-up after endovascular treatment. AJNR Am J Neuroradiol 2010; 31: 1197-1205.

15) Szikora I, Marosfoi M, Salomváry B, et al: Resolution of mass effect and compression symptoms following endoluminal flow diversion for the treatment of intracranial aneurysms. AJNR Am J Neuroradiol 2013; 34: 935-939.

16) Moon K, Albuquerque FC, Ducruet AF, et al: Resolution of cranial neuropathies following treatment of intracranial aneurysms with the Pipeline Embolization Device. $J \mathrm{Neu}$ rosurg 2014; 121: 1085-1092.

17) Binyamin TR, Dahlin BC, Waldau B: Resolution of third nerve palsy despite persistent aneurysmal mass effect after flow diversion embolization of posterior communicating artery aneurysms. J Clin Neurosci 2016; 31: 207-209. 